\title{
Determination of Freeway Space in Completely Edentulous Patients Using Lateral Profile Photographs- In Vivo Study
}

\author{
Monika Dagdiya ${ }^{1} \quad$ Ashok Pakhan ${ }^{1} \quad$ Surekha Dubey ${ }^{1}$ \\ ${ }^{1}$ Department of Prosthodontics, Sharad Pawar Dental College, \\ Datta Meghe Institute of Medical Sciences, Wardha, Maharashtra, \\ India
}

\author{
Seema Sathe ${ }^{1}$ Manish Dagdiya ${ }^{1}$
}

Int J Recent Surg Med Sci 2019;5:6-9

\begin{abstract}
Address for correspondence Monika Dagdiya, Department of Prosthodontics, Sharad Pawar Dental College, Datta Meghe Institute of Medical Sciences, Wardha 442004, Maharashtra, India (e-mail: monica.dagdiya@gmail.com).
\end{abstract}

\begin{abstract}
Keywords

- complete denture

- freeway space

- gonia angle

- lateral profile

photograph

Objective This study aims to determine the freeway space (FWS) using lateral profile photograph (LPP) as an adjunct in completely edentulous patients.

Materials and Methods In this study, 30 patients in the age group of 45 to 60 years who met with the criteria were included. LPPs were taken for all participants using standard protocol and a duplicate copy was obtained, on which three soft tissue reference points-porion (Stp), gnathion (Stgn), and gonion (Stg)-were marked and joined to form an angle Stp-Stg-Stgn. This angle was correlated with the mean FWS obtained using swallowing. Simple linear regression model was used to develop a prediction formula for FWS using Stp-Stg-Stgn angle as the independent variable.

Result This study shows that the angle Stp-Stg-Stgn had a significant negative correlation with FWS.
\end{abstract}

\section{Introduction}

Rest position has been defined as the neutral position attained by the mandible as it is involuntarily suspended by the reciprocal coordination of the elevator and depressor masticatory muscles with the maxillary and mandibular teeth separated. ${ }^{1}$

The provision of freeway space (FWS) in the design of full dentures has long been recognized as one of the chief prerequisites for comfort and optimum function. FWS can be expressed as a gap that occurs in the vertical dimension, separating the position that the mandible assumes in space when it is at rest and its spatial position when the teeth are brought into contact. The problem that arises when the natural teeth are extracted is that the upper border of this gap is lost, and that in designing dentures to replace the natural teeth, the precise level at which this lost dimension previously occurred must somehow be redivined. ${ }^{1}$

The establishment of rest dimension is of first consideration in all types of restorative dentistry. Vertical dimension means the anatomic balance of the mandible. The mandible must not be in the most retruded position because this is a strained position. The proper positioning

received

November 22, 2018

accepted

December 30, 2018 of the condyle heads cannot be accomplished by forcing the mandible to its most retruded position or by forcing it to any other point. The intermaxillary distance proved the same when examined at long intervals. There is no trouble in getting the exact, true relation of the jaws at rest in any individual case. Although the patient may have lost his former jaw movement, he always has his natural rest position. Muscles fatigued by chewing cause the mandible to return to centric occlusion. The patient is instructed to close until he feels natural and comfortable. ${ }^{2}$

The amount of FWS in any individual is mainly an expression of muscle function, its equilibrium, and gravity. When the tension created by the muscles of mastication that elevate the mandible above is in equilibrium with the tension of the musculature that depresses the mandible from below and gravity, then the mandible will be theoretically at rest. However, it is also reported that the FWS does not coincide with the minimal muscle activity. Thus, with the exclusion of muscular activity as a means of determining the extent of FWS, the only alternative is the identification of another anatomical feature, whose nature will also be subject to modification by the effect of the muscle force and unaffected by the changes caused by loss of teeth., 
Though FWS seems easily accessible, it is one of the controversial topics in prosthetic dentistry. It is the basic introductory step in jaw relations on which further complicated ones, such as vertical dimension and centric relation, are contingent. Despite this, determining the level of occlusal contact in full denture cases has received some attention in the literature, and various methods have been put forward from time to time as to how best this might be achieved Potgieter $(1983)^{1}$ compared four of these methods. These were as follows:

- The "phonetic" method described by Morrison (1959), which makes use of the "closest speaking space."

- The method advocated by Shanahan (1956), using the upward thrust which the mandible makes during swallowing.

- The adjustable screw of Timmer (1967) and Lytle (1964) that relies upon the patient's subjective sensibilities in re-creating the position.

- The gauging of biting power through the use of the "biometer," as advocated by Boos (1940).

As proposed by Wolff's law, the muscular functional forces not only influence the FWS but also are responsible for shaping of the internal bone. Based on this concept, the correlation between FWS and structure of the lower facial skeleton has been studied. One study done on the relationship with the form of the lower face found a negative correlation of FWS with the anterior lower facial height and positive with the posterior lower facial height, whereas another cephalometric study done on the relationship with the gonial angle found a statistically significant negative correlation. Thus, the only anatomical feature proved to correlate with FWS and confirm to all the requirements is the gonial angle of the mandible. As the soft tissue profile of an individual depends on the bony structure, this study was planned to evaluate the correlation of the FWS and corresponding soft tissue gonial angle formed on the lateral profile of edentulous individuals in photographs. ${ }^{5}$

\section{Materials and Methods}

Ethical clearance for this study was obtained from the institutional ethics committee. The participants within the age range of 45 to 60 years with class I ridge relation, no acute or previous temporomandibular disorder, were selected from Datta Meghe Institute of Medical Sciences, Sawangi Meghe, (Wardha, Maharashtra, India). Out of the screened 150 potential participants, 30 met the criteria and written consent was obtained from them, before inclusion in this study.

\section{Lateral Profile Photography}

Lateral profile photographs (LPPs) were taken following the standards of extraoral photography using Nikon SLR camera (-Fig. 1).

The participants were instructed to sit straight with their head in natural position achieved by asking them to look into the mirror hung on the wall at their eye level.

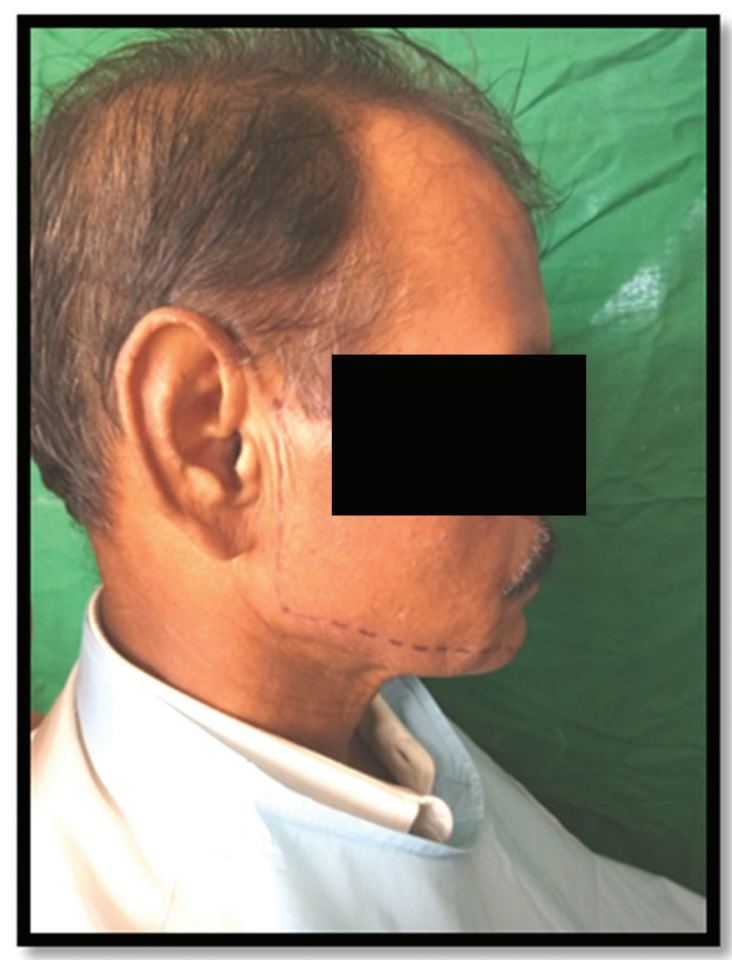

Fig. 1 Lateral profile photograph of the participant.

After confirming that the line drawn from the outer canthus of the eye and upper edge of the ear is parallel to the floor, photographs were taken from a distance of $5 \mathrm{ft}$.

\section{Soft Tissue Reference Points}

Three soft tissue points were considered in this study (-Fig. 2):

1. Point corresponding to porion (Stp): The most superiorly positioned point on the external opening of the auditory meatus.

2. Skin gnathion (Stgn): The point where the anterior curve in the outline of the chin merges into the body of the mandible

3. Gonion (Stg): A point on the curvature of the angle of the mandible formed by the ascending ramus and the inferior border of the mandible.

After which duplicate photo was obtained.

1. To standardize, the photographs were converted by cropping them to size 8 in $\times 10$ in.

2. The images were evaluated, and the gonial angle formed (Stp-Stg-Stgn) was measured ( - Fig. 3 ).

3. To check the reliability of the obtained data, lateral profile analysis, and FWS determination were repeated for five randomly selected participants, by the same investigator and by another investigator.

\section{Observations and Results}

All the data were recorded, tabulated, and subjected to statistical analysis. Statistical analysis was done using SPSS 17.0 software (version 17.0, SPSS, Chicago, Illinois, 


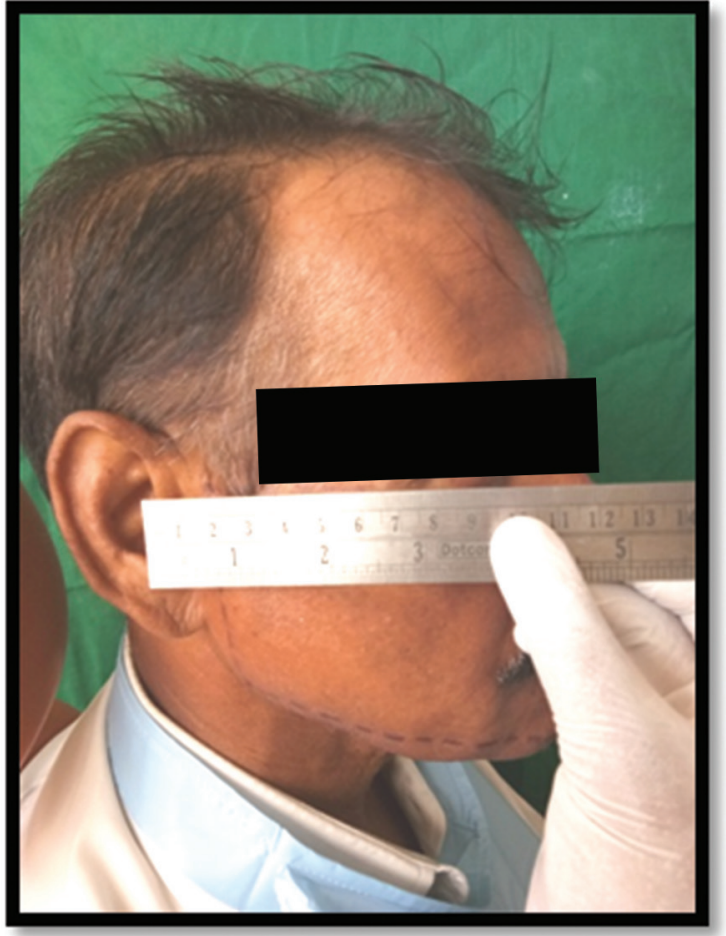

Fig. 2 Marking points Stp-Stg-Stgn.

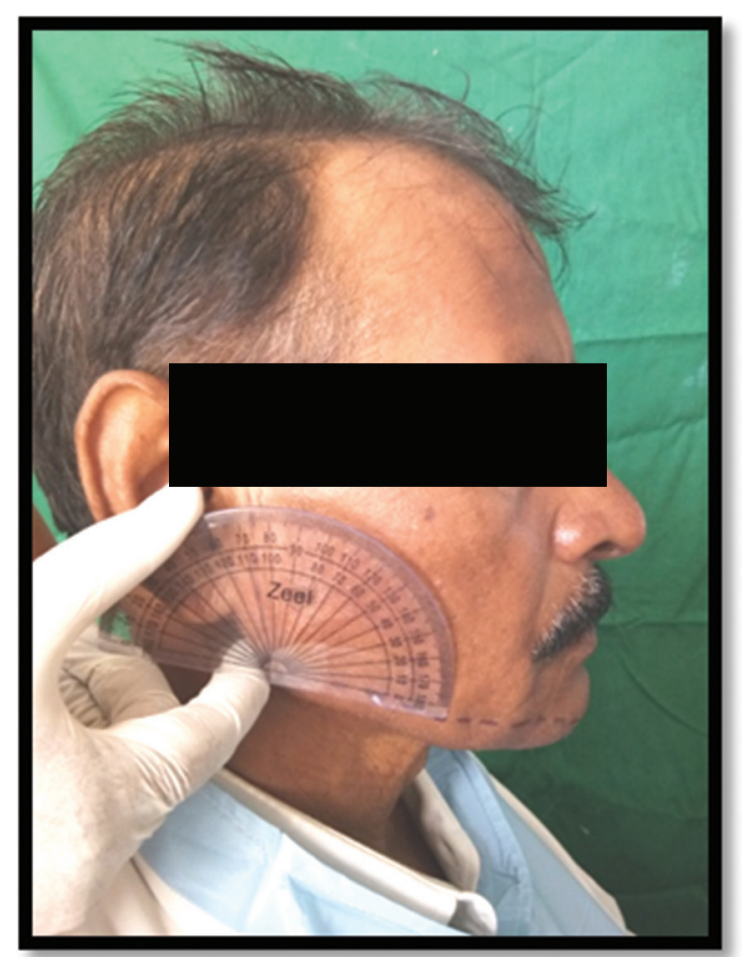

Fig. 3 Gonial angle.

United States). The mean Stp-Stg-Stgn angle was found to be 126 degrees whereas the mean value of FWS obtained through swallowing, phonetics, and no command methods was $2.606 \mathrm{~mm}$ (-Table 1). Reproducibility of the angle Stp-Stg-Stgn on the face was tested using Pearson's correlation test. The correlation between the angle, Stp-Stg-Stgn, and FWS was also determined using Pearson correlation test ( - Table 2).
Table 1 Mean value

\begin{tabular}{|l|l|l|}
\hline & Freeway space & Gonial angle \\
\hline Mean value & $2.606 \mathrm{~mm}$ & 126 degrees \\
\hline
\end{tabular}

Table 2 Pearson's correlation coefficient between freeway space and gonial angle

\begin{tabular}{|l|l|l|l|}
\hline \multicolumn{2}{|l|}{ Correlations } & $\begin{array}{l}\text { Freeway } \\
\text { space (mm) }\end{array}$ & $\begin{array}{l}\text { Gonial angle } \\
\text { (degree) }\end{array}$ \\
\hline \multirow{2}{|c|}{$\begin{array}{l}\text { Freeway } \\
\text { space } \\
\text { (mm) }\end{array}$} & $\begin{array}{l}\text { Pearson } \\
\text { correlation }\end{array}$ & 1 & -0.099 \\
\cline { 2 - 4 } & Sig. (2-tailed) & & 0.601 \\
\cline { 2 - 4 } & $N$ & 30 & 30 \\
\hline \multirow{2}{*}{$\begin{array}{l}\text { Gonial } \\
\text { angle } \\
\text { (degree) }\end{array}$} & $\begin{array}{l}\text { Pearson } \\
\text { correlation }\end{array}$ & -0.099 & 1 \\
\cline { 2 - 4 } & Sig. (2-tailed) & 0.601 & 31 \\
\cline { 2 - 4 } & $N$ & 30 & \\
\hline
\end{tabular}

Simple linear regression analysis was performed for predicting the FWS from the considered angle. The formula used was FWS (in millimeters) $=11.405-0.072 \times($ Stp-Stg-Stgn angle in degrees). ${ }^{6}$ Results were obtained, and graph was plotted (-Fig. 4). Significant values were obtained.

\section{Discussion}

The gonial angle was believed to correlate with the function and shape of the muscles of mastication, mainly masseter and medial pterygoid; these muscles insert into the region of the gonial angle and influence the shape of the mandibular base. As the contractile force of these muscles increases, the angle will be more acute, and the lesser the force, the more obtuse it will be. Thus, studies proved that strong muscle activity correlates with the small anterofacial height and small gonial angle. ${ }^{7.8}$ FWS was described in two ways: adaptive and true FWS. The space that exists when the patient is instructed to voluntarily allow the jaw to relax is the adaptive space, whereas the one present after the relaxation of the masticatory musculature is the true FWS. However, the clinical rest position was not coincident with minimal muscle activity as proved in electromyographic and kinesiographic studies. ${ }^{9}$ FWS is affected by many factors; opening the mouth for an extended period of time, as it happens during our clinical procedures and chewing hard substances can have a profound effect. The muscles that control the mandible can also become tense when any type of mechanical recording device is placed in the mouth or on the head.

Thus, cephalometry was proposed to be an effective method, as it uses specific and predetermined point of bone references to obtain exact measurements. ${ }^{1}$ However, they have certain drawbacks such as picture deformations, superposition of structures, inaccuracy of cephalometric tracing, and also radiation exposure. This highlights the necessity to evaluate for some easier ways that help record the relations without any inconvenience for the patient, as done in this study with profile photographs 


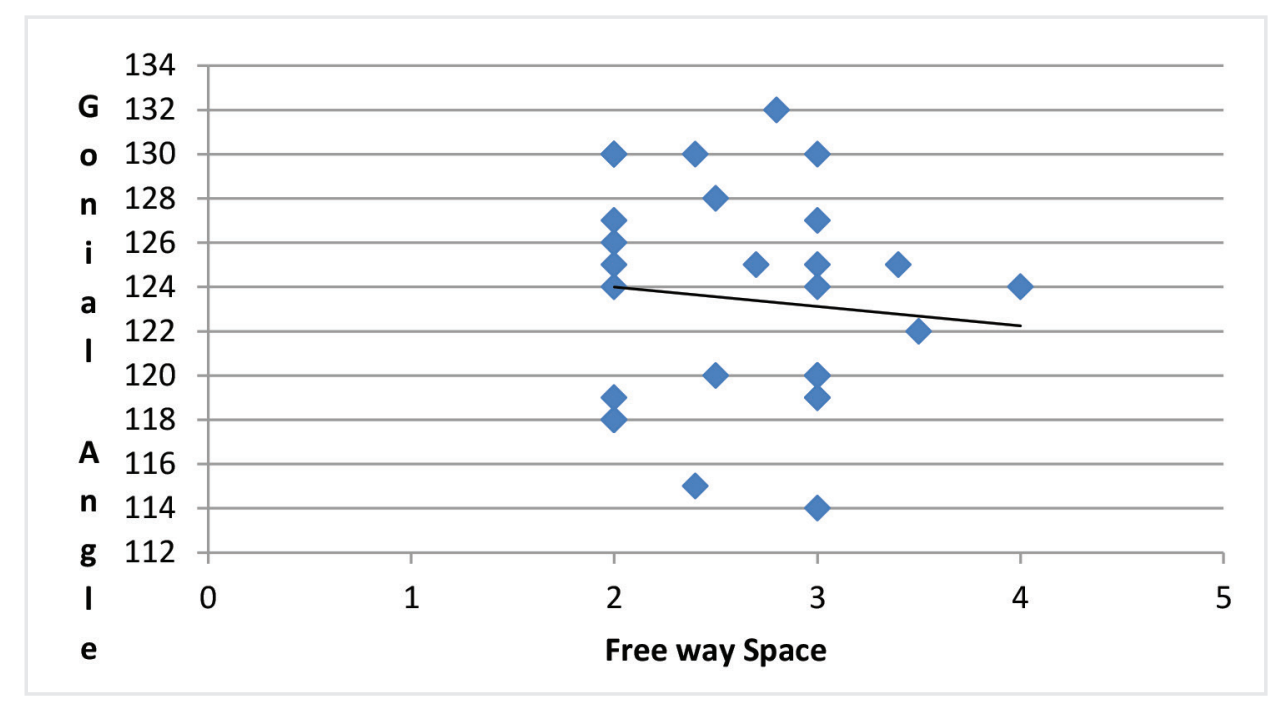

Fig. 4 Scatter diagram between gonial angle and freeway space.

avoiding the aforementioned problems. By following the exact protocol, standard appearance can be retained with simple reproduction of a photograph in the same manner at different time intervals. ${ }^{10}$ Even minor variations in photography have minimal effect on the points and angles in a photograph as observed in the reliability values of this study. The variation in the angle was in the range of 2 to 3 degrees, which had a negligible effect on the prediction of FWS. The chubbiness in the cheek area was also not a problem in our study; the point gonion could be easily palpated without any difficulty. A very strong negative correlation was observed between FWS and the considered angle on the soft tissue profile, which encourages the usage of this method for the determination of the FWS. Though there was a good correlation between the predicted and measured values; on using the formula for Stp-Stg-Stgn angles $>140$ degrees, a small value for FWS will be calculated. Hence, for angle calculations $>140$ degrees, more conventional methods to calculate FWS are recommended additionally. The concept of using Stp-Stg-Stgn angle for the determination of FWS can be generalized to all the population.

\section{Conclusion}

Within the limitations of this study, Stp-Stg-Stgn angle correlated well with the FWS, and hence this new method can be recommended along with other standard ones. Additional studies are also required in this area on edentulous patients. Further studies on photographs as a means of determining the lost facial dimensions can be advocated.

\section{Conflict of Interest}

None declared.

\section{References}

1 Potgieter PJ, Monteith BD, Kemp PL. The determination of freeway space in edentulous patients: a cephalometric approach. J Oral Rehabil 1983;10(4):283-293

2 Gillis R. Establishing vertical dimension in full denture construction. J Am Dent Assoc 1941;28:430

3 Eriksson PO. Muscle-fibre composition of the human mandibular locomotor system. Enzyme-histochemical and morphological characteristics of functionally different parts. Swed Dent J Suppl 1982;12(Supp):1-44

4 Moller E. Evidence that the rest position is subject to servo-control. In: Andreson DJ, Matthews B, eds. Mastication. Bristol, UK: John Wright and Sons; 1976:72

5 Wolff J. Blakiston's New Gould Medical Dictionary. 1st ed. Philadelphia, PA: Blakiston; 1949

6 Vinnakota DN, Kanneganti KC, Pulagam M, Kumar P, Reddy K. Freeway space determination using lateral profile photographs: a pilot study. J Indian Prosthodont Soc 2016;16(3):242-247

7 Huumonen S, Sipilä K, Haikola B, et al. Influence of edentulousness on gonial angle, ramus and condylar height. J Oral Rehabil 2010;37(1):34-38

8 Orthlieb JD, Laurent M, Laplanche O. Cephalometric estimation of vertical dimension of occlusion. J Oral Rehabil 2000;27(9):802-807

9 Konchak PA, Thomas NR, Lanigan DT, Devon RM. Freeway space measurement using mandibular kinesiograph and EMG before and after TENS. Angle Orthod 1988;58(4):343-350

10 Anic-Milosevic S, Slaj M, Lapter-Varga M. Basic principles for taking extraoral photographs. Acta Stomatol Croat 2005;39:201-204

\section{Limitations}

- Picture deformations

- Superposition of structures

- Radiation exposure 